\begin{tabular}{|l|l|c|c|}
\hline Eiszeitalter u. Gegenwart & $\mathbf{2 7}$ & $\begin{array}{c}189-192 \\
2 \mathrm{Abb} .\end{array}$ & Öbringen/Württ. 1976 \\
\hline
\end{tabular}

\title{
Aktive Frostmusterung in Augsburg
}

\author{
Marianne Rolshoven*) \\ Patterned ground (macrotype), middle latitude climate of the high mountains, \\ Augsburg (South Germany).
}

$\mathrm{Kurzf}$ ass ung: Aus Augsburg wird eine quasi-natürliche Bodenmusterung beschrieben, die sich nach Größe und Deutlichkeit der Ausprägung von den bis jetzt im temporär rezent-periglazialen Bereich bekannten Miniaturaufpressungsformen unterscheidet und sich den arktisch-alpinen Bildungen nähert. Ihre Genese hängt von dem spezifischen Standortmilieu der Fundstelle ab.

\section{[Active Patterned Ground at Augsburg]}

A bstract: Patterned ground of macrotype has been found at Augsburg (South Germany). By dimension and distinctiveness of shape these structures differ from those microforms which, since now, seemed to represent the only type of frost phenomena in the region off the recent (climatic) periglacial area.

Aktive Strukturbodenbildung ist in der Vergangenheit häufiger aus Gebieten außerhalb des rezent-periglazialen Bereichs beschrieben worden (WASHBURN et al. 1963, RoHDENBURG \& WALTHER 1968, STINGl 1971, Bild 7 aus dem Jahr 1964), und jeder aufmerksame Beobachter wird zumindest Ansätze zur Bodenmusterung bei lückenhafter oder fehlender Pflanzendecke bereits nach kurzperiodischen Frostwechseln gerade in den Übergangsjahreszeiten finden können. Bisher sind ausschließlich Miniaturformen, Erdknospen und Steinringe, die sich teilweise zu Miniatursteinnetzen zusammenschließen, bekannt geworden.

Entgegen dem bisherigen Kenntnisstand beschränkt sich das aktuelle Musterformeninventar außerhalb des rezent-periglazialen Bereichs nicht allein auf Miniatursteinringe. Dies zeigte sich in Augsburg, wo seit Herbst 1974 Großformen auf dem Flachdach im 14. Stockwerk eines Gebäudes der Universität beobachtet wurden. In pleistozänen Schottern (Fraktion Mittelkies), die durch tonige Beimengungen verunreinigt sind, lassen sich in Abhängigkeit von der Substratzusammensetzung zwei Arten der Strukturierung unterscheiden. Dort, wo Feinmaterial konzentriert auftritt, bilden sich Steinringe mit Innenfelddurchmessern, die bis zu $20 \mathrm{~cm}$ betragen (Abb. 1). Die Rahmenbreite liegt bei $10 \mathrm{~cm}$. Rahmensteine mit einem maximalen Volumen von $60 \mathrm{~cm}^{3}$ werden von der tangentialen Einregelung erfaßt. Im homogenen Grobmaterial ordnen sich die Schotter konzentrisch einem Zentralschotter zu. Es entstehen Steinrosetten, die sich wie die Steinringe zu Netzen zusammenschließen (Abb. 2). Nicht allein charakteristische Einregelung, sondern auch andere Indizien weisen eine frostwechselgesteuerte Genese nach. Nach Frösten kann ein hoher Anteil kantengestellter Schotter bestimmt werden. Sowohl die eigentlichen Feinmaterialzentren als auch die Innenfelder der Rosetten sind dann besonders stark aufgewölbt. Die petrographische und korngrößenspezifische Homogenität des Materials macht eine exakte Aussage über Entmischungsbereiche und Tiefe der von der Sortierung erfaßten Schichten unmöglich. Im Profil scheint nach Aussage der Einregelungsmessungen bei einer Gesamtschottermächtigkeit von $10-12 \mathrm{~cm}$ die Musterungstiefe auf die oberen Lagen

*) Anschrift des Verfassers: Dr. Marianne Rolshoven, Uhlandstraße 60, 8900 Augsburg. 


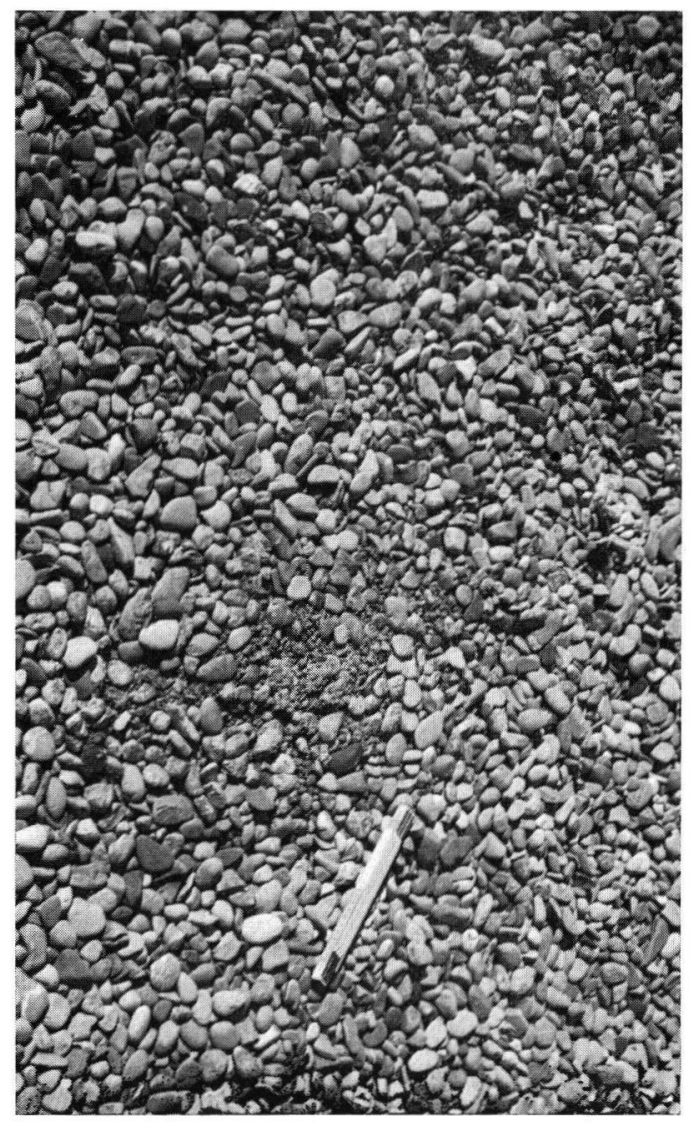

Abb. 1: Quasi-natürliches Steinringnetz in pleistozänen Flußschottern (Standort: Flachdach eines Hochhauses, Maßstab: Zollstock, Datum der Aufnahme: 25. 10. 1976).

beschränkt (bis etwa $5 \mathrm{~cm}$ ). Es kann deshalb nicht eindeutig entschieden werden, ob diese Bodenmusterung durch Sortierung i. S. von Meinardus (1912) oder wie die Miniaturformen infolge Feinmaterialaufpressung durch ein geringmächtiges Steinpflaster entsteht.

Ausbildung und Physiognomie von Miniatursteinringen und Erdknospen hängen direkt von der Schichtung des Substrats ab. Die Deutlichkeit der Musterung ist dann optimal, wenn im Liegenden homogenes bindiges (frostempfindliches) Feinmaterial und im Hangenden eine dünne Grobmaterialauflage vorhanden ist. Da solche Voraussetzungen besonders häufig nach künstlichen Erdbewegungen (Wegebau, Kanalarbeiten etc.) gegeben sind, läßt sich dieser Typ der vorwiegend quasi-natürlichen Bodenmusterung i. a. unter zeitlichem Aspekt exakt erfassen. Die Formen bilden sich spontan als kombinierter Effekt von Tonquellung und frostdynamischem Auffrieren. Sie sind empfindliche Indikatoren kurzperiodischer, flachgründiger Frostwechsel. Ebenso rasch, wie sie entstehen, vergehen sie, etwa als Folge von Starkregen oder nach der Schneeschmelze. Die erwähnten Eigenschaften nähern diese ephemäre Musterung den subtropischen sporadischen Miniaturformen, die u. a. BARTELs (1973) von den Kanaren schildert. Bei entsprechenden frostklimatischen, hydrologischen und edaphischen Voraussetzungen treten Miniaturformen im rezentperiglazialen Bereich neben die eigentlichen Strukturböden als Sortierungsformen. Auf- 


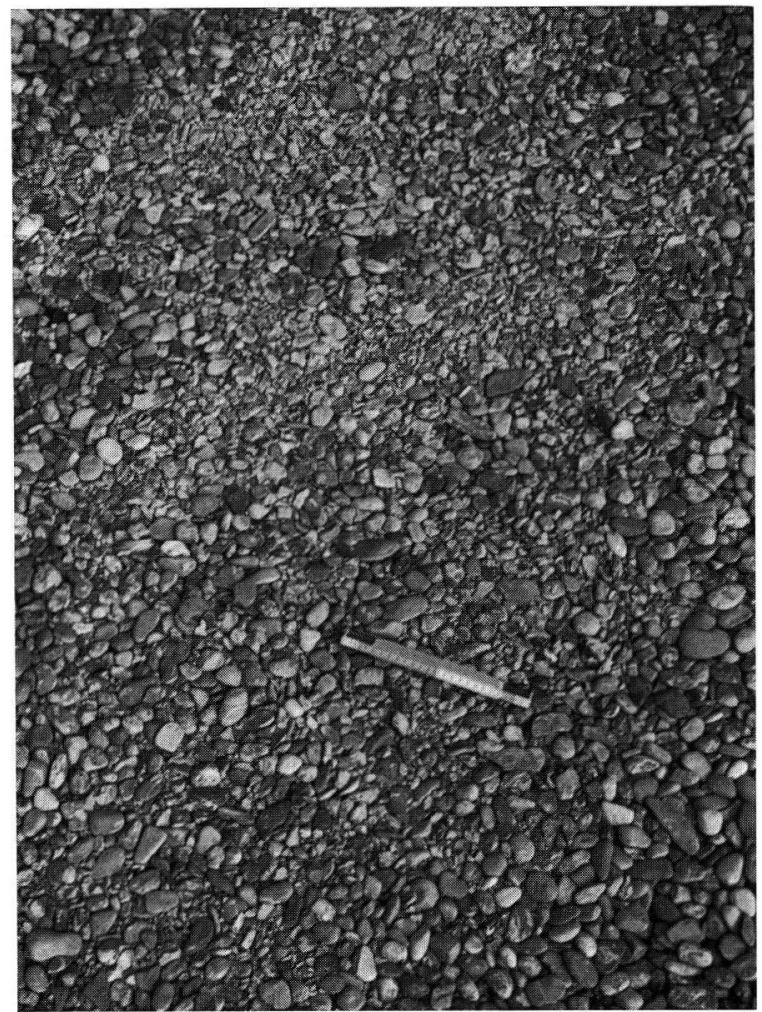

Abb. 2: Feinmaterialzentren neben Verwirbelungen in homogenem Grobmaterial (Steinrosetten). Standort: Flachdach eines Hochhauses, Maßstab: Zollstock, Datum der Aufnahme: 25. 10. 1976.)

pressungsformen können auf Steinringgroßformen als Sekundärmuster auftreten, so daß in der Hochgebirgsstufe der Mittelbreiten eine enge Verzahnung der genetisch unterschiedlichen Typen sporadischer und permanenter Bodenmusterung erfolgt.

Im Gegensatz zu den Miniaturformen sind die in Augsburg nachgewiesenen Großformen nicht instabil. Witterungseinflüsse wirken sich auf die zum größten Teil im Grobmaterial angelegte Strukturierung nicht aus. - Bioturbation kann, lagebedingt, ausgeschlossen werden.

Bei der Genese der Musterböden hat die einfache Tonquellung, die durch zeitweiligen Wasserstau in der Schotterauflage der ebenen Betondachfläche gefördert wird, sicherlich Bedeutung. Die Volumenvergrößerung der Tonminerale spielt ebenso bei der Bildung der alpinen Strukturböden eine Rolle (VoRndRan 1972) wie bei der Entstehung der Mikroformen. Die eigentliche formendifferenzierende Wirkung kommt jedoch der frostdynamisch bedingten Volumenveränderung im Substrat zu. Dies gilt sowohl für die Aufpressung des homogenen Feinmaterials bei der Miniaturformengenese als auch bei den eigentlichen Sortierungsformen. Im Gegensatz sowohl zu den alpinen Strukturböden als auch zu den Miniaturformen ist der Anteil kolloider Bodenbestandteile im geschilderten Fall außerordentlich gering (unter $10 \%$ ). Die Formengröße übersteigt die der Miniaturbildungen beträchtlich. Das Volumen der von der Musterung erfaßten Gesteinsfragmente verhält sich im Vergleich zu den Mikroformen wie Mittelkies zu Feinkies. Beobachtungen aus 
dem rezent-periglazialen Bereich scheinen darauf hinzudeuten, daß das Raumgewicht der von der Musterung erfaßten Klastika als Gradmesser frostdynamischer Aktivität gelten kann.

Charakteristisch für das Gebiet außerhalb des rezenten Periglazialbereichs sind Mikroformen. Dies bestätigen Versuchsanordnungen in Gärten sowohl in Köln (1972/1973) als auch in Augsburg (1975), die bereits nach wenigen Frostwechseln Erdknospen und Andeutungen von Miniaturzentren zeigen, doch Grobmaterial über der Fraktion Feinkies wurde nicht mehr bewegt. Die Entstehung der hier betrachteten Formen muß daher auf spezifische lokale Bedingungen auf dem Flachdach zurückgehen. Der Betondecke mit der geringmächtigen Schotterlage scheint ein Verstärkungseffekt zuzukommen, wie er den azonalen Strukturbodenformen der Gletschervorfelder (Steinringe mit Eiskern bzw. Felskern) eigen ist (KINZL 1928). Aus dieser Verwandtschaft läßt sich auch das Mißverhältnis zwischen relativ großem Formendurchmesser und geringer Mächtigkeit des Schotterkörpers herleiten. Bei zonalen Strukturböden - dies haben Fischer (1970) und jüngst STINGLHerrmann (1976) regelhaft nachgewiesen - besteht nämlich eine direkte Abhängigkeit zwischen wachsenden Formengrößen und Tiefe des unterlagernden Materials.

Das Formungsmilieu der Steinringe und Steinrosetten weicht erheblich von dem der Mikroformen ab. Dies gilt einerseits für die lokalklimatischen Gegebenheiten, andererseits für die Substratzusammensetzung. Bei der Anlage der Steinringe und Steinrosetten spielen sicher zufällige lokale Anreicherungen des Feinmaterials eine initiale Rolle für das Ansetzen von Frostdynamik.

Die Musterformen in Augsburg können formal den arktischen bzw. alpinen Formen angenähert werden. Die Differenzierung der Einzelformen (Steinringe und Steinrosetten) hängt hier wie dort von den edaphischen Voraussetzungen ab.

Das Spektrum der Musterformen im außeralpinen Gebiet erweitert sich damit um einen „arktisch-alpinen“ Typ, dessen Auftreten an besondere Gunstlagen gebunden ist. Charakteristisch sind für diesen Typ eine lange formenbewahrende Inaktivitätsphase während der warmen Jahreszeit und eine lange Phase potentieller Aktivität, die vom Herbst bis in das Frühjahr reicht. Damit unterscheiden sie sich von den Musterböden im rezent-periglazialen Bereich, deren Aktivitätsgrad auf eine mit zunehmender Höhe verkürzte Periode sommerlicher Aktivität beschränkt ist.

\section{Schriftenverzeichnis}

Bartels, G. (1973): Jahreszeitlich bedingte Strukturböden auf den Kanaren. - Die Erde, 104 (3-4): 314-319; Berlin.

Fischer, K. (1970): Frostmusterböden in den Dolomiten. - Der Schlern, 44: 136-142; Bozen.

KinzL, H. (1928): Beobachtungen über Strukturböden in den Ostalpen. — Pet. Mitt., 74: 261265; Gotha.

Meinardus, W. (1912): Beobachtungen über Detritussortierung und Strukturböden auf Spitzbergen. - Z. Ges. Erdkde.: 250—259; Berlin.

Rohdenburg, H. \& Walther, D. (1968): Rezente Strukturböden in Gießen. - Eiszeitalter u. Gegenwart, 19: 279-282; Öhringen/Württ.

Stingl, H. (1971): Zur Verteilung von Groß- und Miniaturformen von Strukturböden in den Ostalpen. - Nachr. Akad. Wiss. Göttingen II. Math.-Physikal. K1., 2: 25-40; Göttingen.

- , Herrmann, R. (1976): Untersuchungen zum Strukturbodenproblem auf Island. Geländebeobachtungen und statistische Auswertung. - Z. Geomorph. N. F., 20 (2): 205-226; Berlin.

Vorndran, G. (1972): Kryopedologische Untersuchungen mit Hilfe von Bodentemperaturmessungen. - Münchener Geogr. Abh., 6: 70 S.; München.

Washburn, A. L., Smith, D. \& Goddard, R. (1963): Frost cracking in a middle-latitude climate. - Biuletyn Periglacjalny, 12: 175-189; Lodz. 